\title{
Transcriptome Profiling of Wild Arachis from Water-Limited Environments Uncovers Drought Tolerance Candidate Genes
}

\author{
Ana C. M. Brasileiro ${ }^{1}$ - Carolina V. Morgante ${ }^{1,2}$ • Ana C. G. Araujo ${ }^{1}$. \\ Soraya C. M. Leal-Bertioli ${ }^{1}$ - Amanda K. Silva ${ }^{1}$ - Andressa C. Q. Martins ${ }^{1}$. \\ Christina C. Vinson ${ }^{1} \cdot$ Candice M. R. Santos ${ }^{1,3}$. Orzenil Bonfim $^{1} \cdot$ Roberto C. Togawa $^{1}$. \\ Mario A. P. Saraiva ${ }^{1}$ - David J. Bertioli ${ }^{4}$ Patricia M. Guimaraes ${ }^{1}$
}

Published online: 11 April 2015

(C) The Author(s) 2015. This article is published with open access at Springerlink.com

\begin{abstract}
Peanut (Arachis hypogaea L.) is an important legume cultivated mostly in drought-prone areas where its productivity can be limited by water scarcity. The development of more drought-tolerant varieties is, therefore, a priority for peanut breeding programs worldwide. In contrast to cultivated peanut, wild relatives have a broader genetic diversity and constitute a rich source of resistance/tolerance alleles to biotic and abiotic stresses. The present study takes advantage of this diversity to identify drought-responsive genes by analyzing the expression profile of two wild species, Arachis duranensis and Arachis magna (AA and BB genomes, respectively), in response to progressive water deficit in soil. Data analysis
\end{abstract}

Accession numbers for the GenBank sequence database: JZ390113 to JZ390862

Electronic supplementary material The online version of this article (doi:10.1007/s11105-015-0882-x) contains supplementary material, which is available to authorized users.

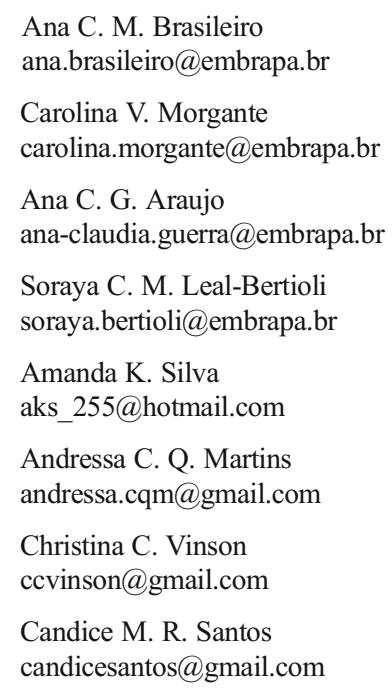

from leaves and roots of $A$. duranensis (454 sequencing) and A. magna (suppression subtractive hybridization ( $\mathrm{SSH})$ ) stressed and control complementary DNA (cDNA) libraries revealed several differentially expressed genes in silico, and 44 of them were selected for further validation by quantitative RT-PCR (qRT-PCR). This allowed the identification of drought-responsive candidate genes, such as Expansin, Nitrilase, $N A C$, and $b Z I P$ transcription factors, displaying significant levels of differential expression during stress imposition in both species. This is the first report on identification of differentially expressed genes under drought stress and recovery in wild Arachis species. The generated transcriptome data, 
besides being a valuable resource for gene discovery, will allow the characterization of new alleles and development of molecular markers associated with drought responses in peanut. These together constitute important tools for the peanut breeding program and also contribute to a better comprehension of gene modulation in response to water deficit and rehydration.

Keywords SSH libraries $\cdot 454$ Sequencing · Differential gene expression $\cdot$ Dry-down $\cdot$ Peanut wild relatives $\cdot$ qRT-PCR

\section{Introduction}

Peanut (Arachis hypogaea L.) is an oilseed crop cultivated worldwide and one of the major grain legumes in tropical and subtropical regions. However, its productivity is strongly affected in drought-prone areas by water scarcity. Drought is one of the most serious constraints to crop production and, associated with the predicted consequences of global climate change, increases the need for drought-adapted varieties. Considering the highly complex aspects of drought tolerance in plants, the understanding of response mechanisms to waterlimited conditions and identification of the pathways and genes involved in these responses are strategic for the development of new varieties (Hu and Xiong 2014; Juenger 2013).

In this respect, transcriptome approaches have the potential to aid the understanding of tolerance mechanisms and to orientate gene discovery. Moreover, high-throughput sequencing allows the study of simultaneous gene expression and their regulation profiles in different biological processes, thus enabling species comparisons and revealing key information regarding evolution divergence and domestication processes $(\mathrm{Li}$ et al. 2012; Madlung and Wendel 2013). Therefore, large transcriptome surveys have been undertaken in recent years for many crop plants, including peanut (Chen et al. 2013; Wu et al. 2013; Yin et al. 2013; Zhang et al. 2012).

Peanut has a large tetraploid genome, and its genetic studies have been hindered over the years by the scarcity of genomic resources (Brasileiro et al. 2014; Holbrook et al. 2011; Pandey et al. 2012) such as physical maps, a comprehensive transcriptome database, and a fully sequenced genome, which is now on the verge of being accomplished by the International Peanut Genome Initiative (IPGI; http://www. peanutbioscience.com/homepage.html). In addition, only recently, studies identifying genes associated with drought stress responses using transcriptome approaches for the genus Arachis have become available (Ding et al. 2014; Govind et al. 2009; Guimaraes et al. 2012; Jain et al. 2001; Li et al. 2014; Luo et al. 2005; Ranganayakulu et al. 2011).

Unlike the cultivated species, wild Arachis is more genetically diverse having undergone selection through evolution to adapt to a wide variety of habitats and conditions and is more likely to harbor resistance/tolerance to biotic and abiotic stresses (Bertioli et al. 2011). In particular, the genus Arachis is endemic to South America being mostly associated with tropical savannah regions, characterized by a marked dry season during the southern winter. In particular, Arachis duranensis and Arachis magna, diploid wild annual species with $\mathrm{AA}$ and $\mathrm{BB}$ Arachis genomes, respectively, are native to low rainfall regions in Bolivia and Argentina (Krapovickas and Gregory 2007; Leal-Bertioli et al. 2012; Robledo et al. 2009). Indeed, previous studies showed that the accession K7988 of A. duranensis and KG30097 of A. magna displayed the most conservative transpiration profile under waterlimited conditions when compared to other genotypes including wild, cultivated, and synthetic amphidiploids Arachis (Leal-Bertioli et al. 2012), making them very interesting genotypes for drought-related gene discovery. Moreover, these two species are also the parentals of the mapping populations used to develop the diploid A and B genetic maps (Moretzsohn et al. 2005, 2009), which are fundamental for the localization of candidate genes in genetic reference maps and co-localization with QTLs (Leal-Bertioli et al. 2009).

In addition, the availability of a comprehensive A. duranensis root transcriptome under drought stress database developed by our group (Guimaraes et al. 2012) directed the selection of $A$. duranensis together with A. magna, as representatives of the AA and BB genomes of Arachis and good candidates to identify drought-responsive genes in the present study. The analysis of deep-sequencing-based transcriptome profiling of both genotypes submitted to gradual soil water deficit enabled the in silico identification of many differentially expressed genes, from which, 44 candidates were selected for further quantitative RT-PCR (qRT-PCR) validation. The data herein constitutes a novel genomic resource for wild Arachis available for the development of new molecular markers and gene discovery, thus facilitating the transfer of important traits into cultivated peanut varieties.

\section{Materials and Methods}

\section{Soil Gradual Water Deficit (Dry-Down) Experiment}

Seeds of A. duranensis (accession K7988) and A. magna (accession KG30097) were obtained from the Embrapa Active Germplasm Bank (Brazil) and germinated in germitex paper with $2 \%(w / v)$ Ethrel (2-chloroethylphosphonic acid) and $0.05 \%(w / v)$ thiram (tetramethylthiuram disulfide). Plantlets were transferred to pots (one per pot) containing $500 \mathrm{~g}$ of soil and kept regularly watered in a greenhouse.

Dry-down experiments were carried out using 3-month-old plants, as previously described (Leal-Bertioli et al. 2012). Briefly, plants were divided into two sets: one submitted to dry-down (drought-stressed (DS) plants), and another, the 
control group, was regularly watered (well-watered (WW) plants). To prevent water evaporation from soil, the pots were bagged, leaving the plant stem and leaves outside the plastic bag. Individual transpiration rate (TR) was estimated gravimetrically, every day, and normalized transpiration rate (NTR) calculated as the ratio between TR values and the average TR for the first 3 days for each individual plant, as described by Muchow and Sinclair (1991). Greenhouse temperature and relative air humidity were recorded hourly using an Electronic Datalogger Sato SK-L200TH II (Sato, Japan).

Leaves and roots of $A$. duranensis and A. magna were collected at different points, based on the average NTR of DS plants, corresponding to different stages of water deficit and re-watering: (i) point 1, beginning of drought stress (NTR values between $0.65-0.60$ ); (ii) point 2 , representing the rapid decline in the TR of DS plants (NTR values around 0.4); (iii) point 3 , considered the endpoint for the water deficit treatment (NTR values below 0.3); (iv) point 4, plant re-watering to $70 \%$ field capacity (FC) followed by sample collection after $30 \mathrm{~min}$ for A. duranensis and $5 \mathrm{~h}$ for A. magna; and (v) point 5, only A. duranensis plants collected $72 \mathrm{~h}$ after re-watering, when DS plants showed a phenotype similar to the WW plants. For both species, samples from leaves and roots of 15 DS plants, and another 15 from WW, were harvested at each collecting point, immediately frozen in liquid nitrogen and stored at $-80{ }^{\circ} \mathrm{C}$.

\section{RNA Extraction}

Extraction of total RNA was conducted using a modified lithium chloride protocol, as previously described (Morgante et al. 2011). Total RNA was further purified on Invisorb Plant RNA Mini columns (InViTek, Berlin, Germany), according to the manufacturer's instructions. RNA quality/ integrity was checked on ethidium-bromide-stained $1.5 \%$ $(w / v)$ agarose gels and quantified using a NanoDrop ${ }^{\circledR}$ ND-1000 spectrophotometer (Thermo Scientific, Waltham, USA). RNA samples were subsequently treated with DNase (TURBO DNA-free ${ }^{\mathrm{TM}}$, Ambion, USA), according to the manufacturer's instructions. Total RNA extracted from A. duranensis plants was used exclusively for sample preparation for qRT-PCR analysis whereas RNA from A. magna plants was used for both complementary DNA (cDNA) libraries construction and qRT-PCR analysis.

\section{cDNA Library Construction and Sequence Analysis}

Sequences from $A$. duranensis cDNA libraries previously obtained by our group (Guimaraes et al. 2012) were used for differential expression analysis and drought-responsive gene mining. The number of raw sequences mapping from each library (WW and DS) to the resulting clusters of de novo transcript assembly (Guimaraes et al. 2012) was used as an estimate of transcript quantity using the "gene counts" model, as previously described (Beneventi et al. 2013). Genes that showed distinct numbers of counts between the two libraries and similarity to drought-stress-responsive sequences in BLASTX algorithm (Altschul et al. 1990) were selected for qRT-PCR analysis.

A. magna subtractive cDNA libraries were constructed using WW and DS leaf RNA samples, assembled at equal amounts from collecting points 1,2 , and 3. PolyA+ RNA was subsequently isolated from $3 \mathrm{mg}$ of each RNA assemblage using Oligotex ${ }^{\circledR}$ kit (Qiagen, Hilden, Germany). Suppression subtractive hybridization (SSH) libraries were produced using PCR-Select ${ }^{\mathrm{TM}}$ cDNA Subtraction Kit (Clontech, Mountain View, USA) in both directions; i.e., cDNA from stressed plants was used as the driver [given rise to stressed (STR) library] and, afterward, as the tester [control (CTR) library]. Subtractive PCR products were sequenced by the Sanger method using an ABI Prism 3700 DNA Analyzer (Applied Biosystems Inc., Foster City, USA). A. magna expressed sequence tags (ESTs) had their quality checked before being trimmed and clustered following Telles and Silva (2001). Functional annotation of A. magna ESTs for known proteins/genes was conducted as for $A$. duranensis (Guimaraes et al. 2012), using the BLASTX algorithm (Altschul et al. 1990) followed by Blast2GO tool for functional category assignment (Gotz et al. 2008).

\section{Gene Validation by qRT-PCR}

A first series of differential expression analysis between DS and WW plants by qRT-PCR used A. duranensis and A. magna samples gathered in pools. For this, leaf and root RNA samples extracted from five individuals at collecting points 1,2 , and 3 were pooled at equal amounts, constituting a biological replicate with 15 samples. For each treatment (DS and WW) and species, three independent replicates were formed. RNA was quality checked and treated with DNase as described above and used for first-strand cDNA synthesis according to Morgante et al. (2011).

qRT-PCR was performed in three technical replicates using the Platinum ${ }^{\circledR}$ SYBR ${ }^{\circledR}$ Green qPCR SuperMix-UDGw/ROX kit (Invitrogen, Carlsbad, USA), on the ABI 7300 Real-Time PCR System (Applied Biosystems, Foster City, USA). Reactions and primer design were conducted as previously described (Morgante et al. 2013). Efficiencies for each primer pair and optimal cycle threshold $(\mathrm{Cq})$ values were estimated using the online real-time PCR Miner tool (Zhao and Fernald 2005). Average Cq values were normalized to two reference genes, for each species, as previously established (Morgante et al. 2011). Expression ratios of messenger RNA (mRNA) transcripts in DS samples relative to WW were determined and statistically tested using REST 2009 v. 1 software (Pfaffl et al. 2002). 
Eight genes were selected as candidates for more detailed expression study using a further series of qRT-PCR analysis. For this, samples from each collecting point and tissue from five individuals were pooled in equal amounts to constitute a biological replicate. Three independent replicates with 15 samples each were thus formed per collecting point (1 to 5), treatment (DS and WW), and tissue (leaf and root). The assembled RNA was treated as described above.

\section{Results and Discussion}

\section{Dry-Down Experiments}

The transpiration profile of $A$. duranensis and A. magna during gradual water deficit experiments is represented by the NTR behavior in Fig. 1. NTR curves corresponding to overall transpiration data obtained by gravimetrical measurements followed, for both wild species, the typical pattern previously described for Arachis and other tropical legume species (LealBertioli et al. 2012; Sinclair and Ludlow 1986; Zaman-Allah et al. 2011). NTR values for both species were adjusted to values close to 1 (Fig. 1) in order to have a similar transpiration behavior for the beginning of the dry-down. With the water deficit imposition, NTRs for DS plants gradually decreased (Fig. 1), reflecting the changes in transpiration patterns, as lower water availability in soil induces the reduction of plant transpiration rates (Sinclair and Ludlow 1986). Therefore, to better capture gene expression modulations associated with the variations in transpiration profile, samples from both species were collected at three time points (collecting points 1 to 3 ; Fig. 1) in an attempt to provide representative stages of limited water stress, as described by Sinclair and Ludlow (1986). Subsequent collecting points (4 and 5) corresponded to DS plant drought recovery stages after soil rehydration to $70 \% \mathrm{FC}$. During the experiments, temperature ranged from 22 to $31{ }^{\circ} \mathrm{C}$ and relative air humidity from 70 to $80 \%$.

A. duranensis drought imposition lasted 11 days. During the stress, transpiration rates of DS plants gradually declined and samples were collected at NTR values of $0.65,0.44$, and 0.28 (Fig. 1). At NTR 0.28 (point 3), A. duranensis DS plants displayed a dehydrated appearance, with wilting leaflets with chlorotic areas and roots with evident desiccation, appearing brownish and brittle whereas WW plants displayed a normal phenotype (Fig. 2a, b). Therefore, point 3 was selected as the point in which the drought condition threshold was reached. Following this point, soil of DS plants was fully rehydrated and samples were collected after $30 \mathrm{~min}$ (point 4) and $72 \mathrm{~h}$ (point 5) of re-watering. At point 5, DS plants recovered transpiration rates close to WW $(\mathrm{NTR}=0.92)$, with leaf and root phenotypes resembling those of controls (Fig. 2c).
A. magna drought imposition lasted 8 days and NTR values of DS plants dropped sharply at the first day of drydown and then declined slightly every day during the subsequent days (Fig. 1). A. magna DS samples were collected at the NTR $0.61,0.42$, and 0.25 (points 1 to 3 , respectively; Fig. 1). At NTR 0.25, established as the threshold point for the imposed stress, only mild symptoms of dehydration in leaves and roots were observed in DS plants compared to WW (Fig. 2d, e). Remarkably, A. magna DS plants recovered the phenotype similar to the WW just $5 \mathrm{~h}$ after re-watering (Fig. 2f).

The different time necessary for each species to reach similar NTR under the gradual water deficit herein imposed (Fig. 1) suggests that specific mechanisms of drought responses or physiological differences could be inherent to each genotype. In fact, this assumption is corroborated by previous results showing that $A$. duranensis and $A$. magna transpiration patterns under drought stress conditions differ from each other, yet with the more "conservative" behavior for water use than other Arachis genotypes (Leal-Bertioli et al. 2012). A. duranensis and A. magna grow in low rainfall areas (Krapovickas and Gregory 2007; Leal-Bertioli et al. 2012), and their conservative transpiration performance under limited water availability might be associated with phenotypic plasticity for adaptation to such condition, typical of their native environment. Along with other physiological mechanisms, linked to adaptive phenotypic plasticity, the variation in transpiration rates potentially allows plant endurance during drought stress conditions (Juenger 2013; Zaman-Allah et al. 2011). Therefore, dry-down experiments enabled a detailed comparative analysis of $A$. duranensis and A. magna transpiration behavior and also an accurate root and leaf sampling during gradual soil water deficit to be used in comprehensive transcriptome analysis.

\section{A. duranensis Sequence Analysis and Functional Annotation}

Transcriptome 454 sequence data of $A$. duranensis roots under water deficit previously produced by this group (Guimaraes et al. 2012) was further investigated in order to identify genes involved in drought stress responses. From the 12,792 high confident consensus sequences (contigs) generated by de novo assembly, 7483 (58 \%) showed significant functional annotation of which $40 \%$ are legume homologous with the highest similarities to Glycine (24\%) and Medicago (7\%) (Fig. 3). This is probably due to the large number of Glycine sequences available in public databases ( $\mathrm{Li}$ et al. 2012) and this being one of the most closely related genus to Arachis among the papilionoid legumes, especially compared to the model legumes Medicago and Lotus (Bertioli et al. 2011). Within species of the genus Arachis, transcript similarity was only $3 \%$ (Fig. 3), reflecting the 


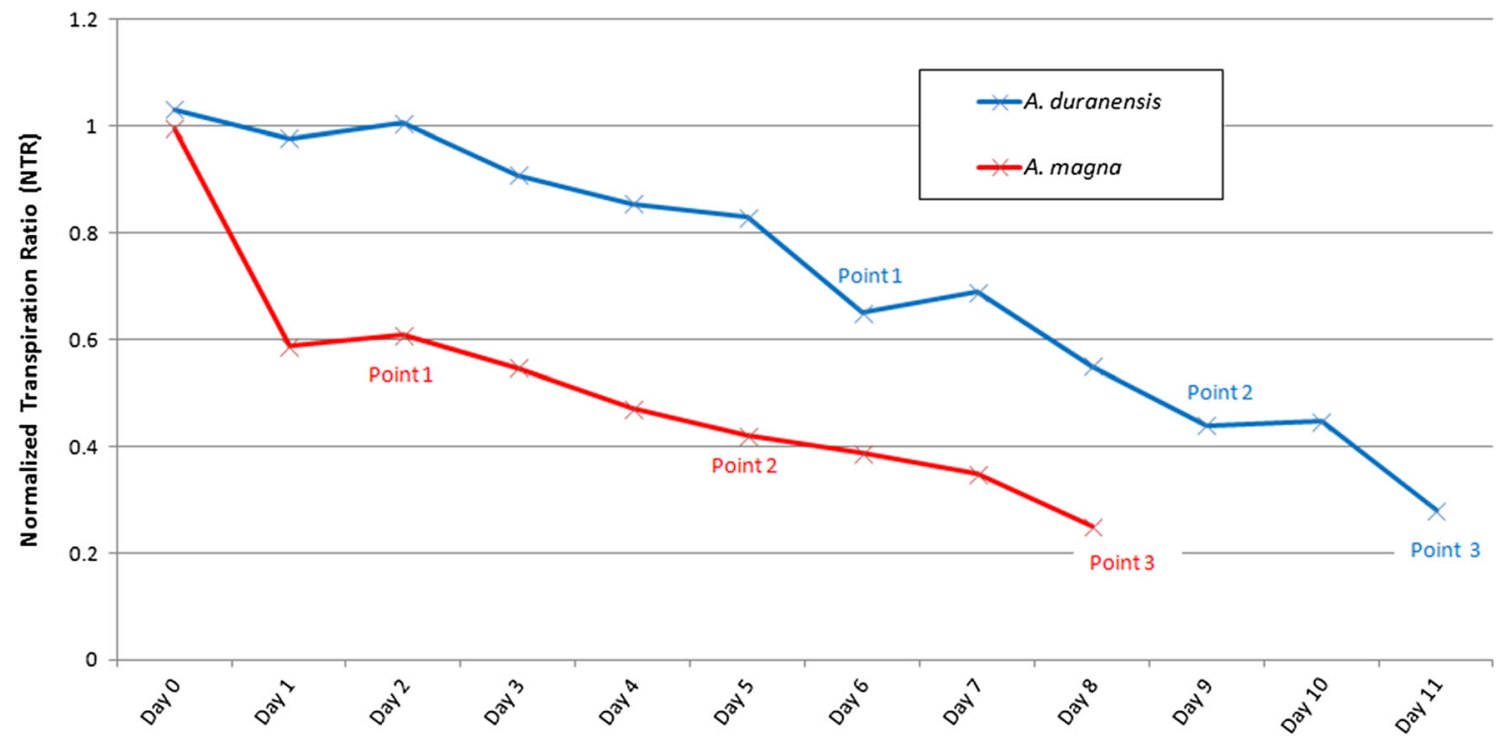

Fig. 1 Normalized transpiration ratio (NTR) of A. duranensis and A. magna during dry-down and after soil re-watering. Day 0 corresponds to dry-down start. Collecting points (1 to 3 ) are showed in blue for A. duranensis and in red for A. magna

relatively low amount of sequence data currently available. Nevertheless, the high degree of sequence homology with peanut suggests a close evolutionary relationship between peanut and wild $A$. duranensis, its most probable donor of the maternal genome (Robledo et al. 2009).
From the $12,792 \mathrm{~A}$. duranensis generated contigs, 5236 (41\%) were exclusively present in the DS library, 2511 $(20 \%)$ only in the WW library, and the remaining 5045 $(39 \%)$ in both libraries. Additional in silico analysis estimated gene abundance per library, using the normalized raw number
Fig. 2 a-f Roots of A. duranensis from two WW plants (a) and from two DS plants (b) collected at point 3 (NTR $0.28)$ and from two DS plants (c) collected at point 5 ( $72 \mathrm{~h}$ after soil re-watering). Each root corresponds to an independent individual. Leaves of A. magna from one WW plant (d) and from one DS plant (e) collected at point 3 (NTR 0.25) and from one DS plant (f) collected at point $4(5 \mathrm{~h}$ after soil re-watering). Each aerial part corresponds to an independent individual. For both species, DS roots (b) and leaves (e) show a phenotype strongly affected by drought imposition when compared to normal WW plants (a, d), such as a dehydrated appearance, roots with evident desiccation, brownish and brittle appearance (b), and wilting leaflets with chlorotic areas (e). The phenotype of re-watered roots (c) and leaves (f) is partially recovered (WW alike) after 72 and $5 \mathrm{~h}$, respectively
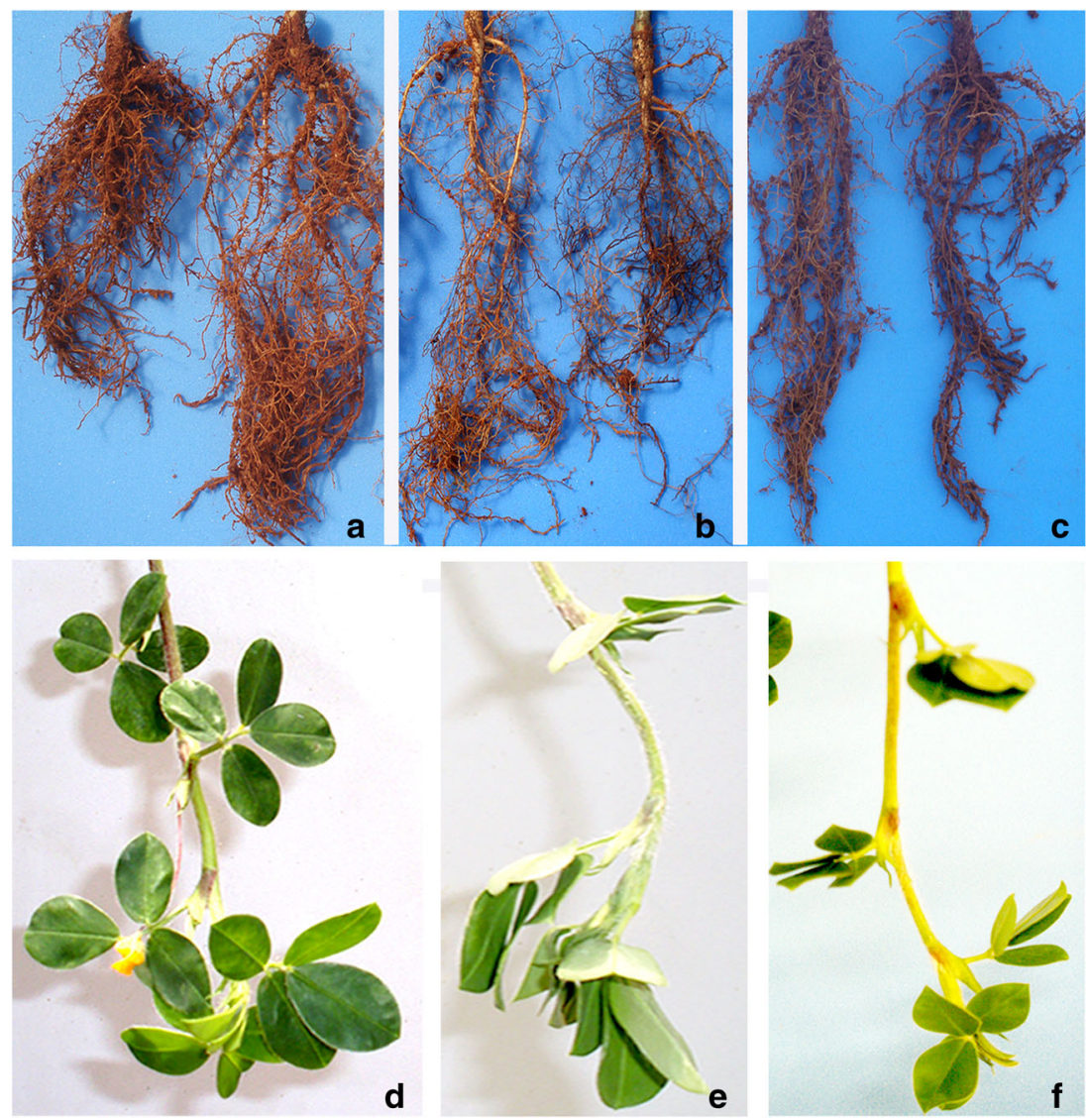
a

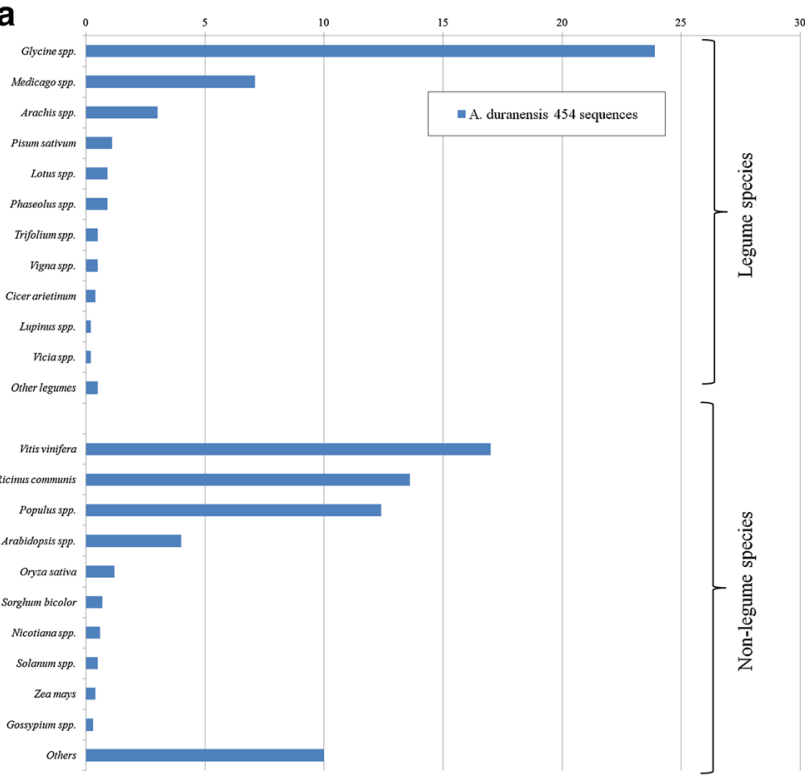

Fig. 3 a, b Distribution by plant species of 12,792 A. duranensis contigs (a) and 757 A. magna unigenes (b), according to BLAST hits. Similarities to plant species are determined and affected by the data available in the

of reads mapped in contigs, according to Beneventi et al. (2013). Normalized read count assignment represents the abundance of each contig in the sample, and it can be suitable for estimation of transcript levels in organisms without reference genome, such as Arachis, when gene models are not known (Jain 2012). In the present analysis, only contigs with over ten reads $(11.8 \%)$ after normalization and with a fold change $>2.0$ or $<-2.0$ were taken into account and ranked according to the number of reads in each library. Based on this, 677 contigs were considered positively regulated under stress condition, as they showed a higher number of reads in the stressed library (26 exclusive) than in the control, while 837 contigs ( 30 exclusive) were negatively regulated, as they were more abundant on the control library. Thirty-one A. duranensis candidate genes with top ranked differentially expressed contigs and which also had a functional annotation related to drought-tolerance were selected for further expression analysis by qRT-PCR. Among them, 18 were associated with drought-responsive genes and 13 to transcription factors (TFs) involved in abiotic stress signaling (Table 1).

\section{A. magna Sequence Analysis and Functional Annotation}

In silico analysis of the two A. magna cDNA libraries revealed 757 high-quality ESTs (Table 2), with an average size of $331 \mathrm{bp}$, which are available in GenBank dbEST (JZ390113 to JZ390862). A. magna ESTs were clustered into 284 unigenes (105 contigs and 179 singletons), with the number of reads per contig ranging from 2 to 56 and a novelty index of $32.8 \%$ (Table 2). Among these unigenes, 194 (68.3\%) had significant BLASTN hits (using default parameters) with at

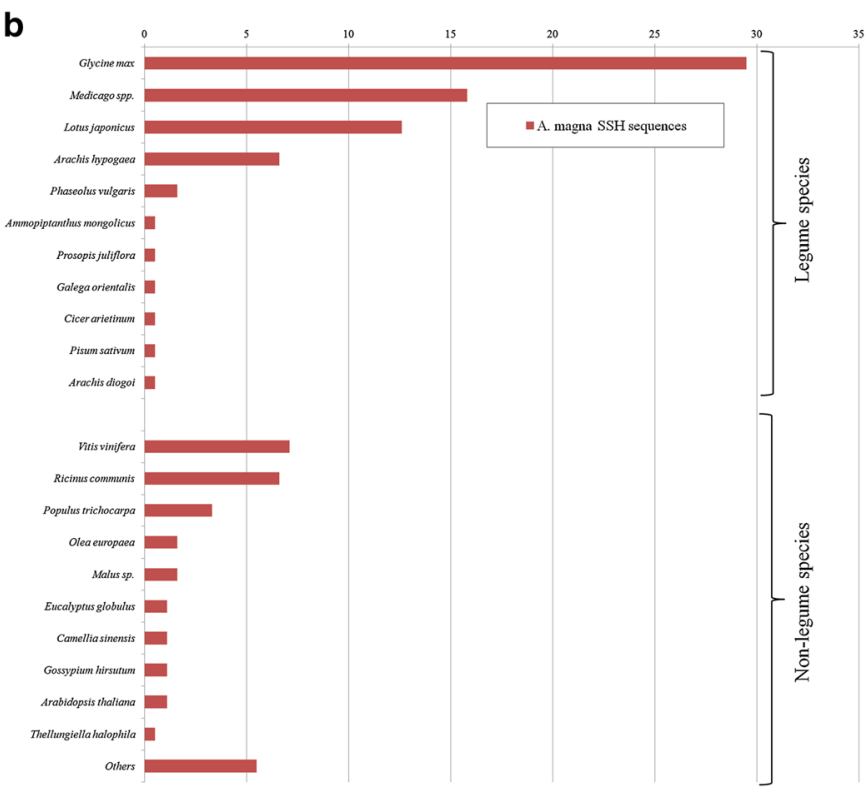

relevant public databases. Bar values correspond to the percentage of unigenes in relation to the total number of annotated sequences of A. duranensis 454 and A. magna $\mathrm{SSH}$ sequences

least one contig present in $A$. duranensis 454 libraries, suggesting that $A$. duranensis and $A$. magna share some of the genes involved in the signaling pathways in response to water deficit. BLASTX and Blast2GO algorithms revealed that approximately half of these sequences $(48 \%)$ had significant matches to genes encoding proteins with known functions, in accordance to previous Arachis transcriptome studies on peanut or AA genome Arachis species (Ding et al. 2014; Govind et al. 2009; Guimaraes et al. 2012; Guo et al. 2008; Li et al. 2014; Proite et al. 2007; Ranganayakulu et al. 2011; Tirumalaraju et al. 2011). Therefore, these $A$. magna unigenes represent the first functionally annotated transcriptome analysis for a BB genome of an Arachis species.

The identified genes with known function could be then assigned to three broad functional categories, i.e., biological process, cellular component, and molecular function (Fig. 4). The biological process category "metabolic process" was the most prevalent ( $28 \%$ of sequences), followed by "cellular process" (20\%) and "response to abiotic stimulus" (10\%). In the cellular component category, genes assigned as "organelle part" accounted for the largest group (27\%), followed by "chloroplast" (16\%), "membrane" (15\%), and "mitochondrion" (14\%). For molecular function, the most abundant sequences were associated with "catalytic activity" (16\%), "binding" (12\%), followed by "nucleotide binding" (10\%). Based on GO annotation, A. magna ESTs associated to the response to water deprivation and to signal transduction pathways under drought, such as lipocalin, LEA, and dehydrin, could also be identified.

The majority of the A. magna annotated sequences (70\%), as for A. duranensis, had the best BLASTX hits with legume 


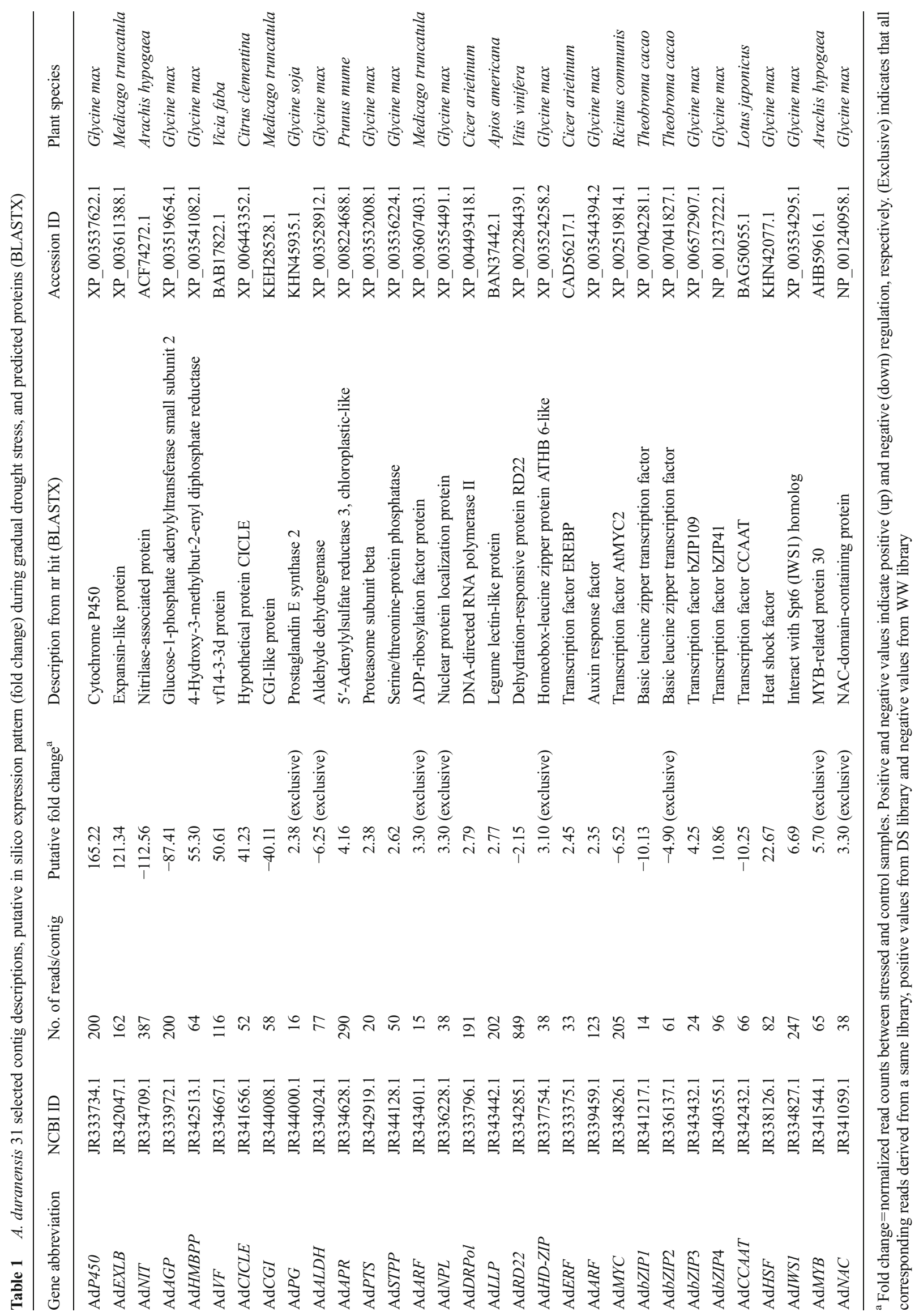


Table 2 In silico analysis of the suppression subtractive hybridization (SSH) sequences from A. magna libraries

\begin{tabular}{llrrrr}
\hline SSH libraries & Number of ESTs & Number of unigenes & Singletons & Contigs & Novelty index (\%) \\
\hline Stressed library subtracted from control library (STR) & 594 & 246 & 159 & 87 & 38.96 \\
Control library subtracted from stressed library (CTR) & 163 & 38 & 20 & 18 & 21.96 \\
Stressed and control libraries & 757 & 284 & 179 & 105 & 32.80 \\
\hline
\end{tabular}

transcripts, mostly with Glycine (30\%), Medicago (16\%), Lotus (13\%), and Arachis (7.1\%) (Fig. 3). Interestingly, subtracted expressed genes of A. magna also showed similarity to other legumes, such as Ammopiptanthus mongolicus and Prosopis juliflora, and to the non-legume Thellungiella halophila (Fig. 3), which are emerging as model species for drought and salt tolerance studies (Sawal et al. 2004; Volkov and Amtmann 2006; Zhou et al. 2012).

Among the $105 \mathrm{~A}$. magna contigs identified, 87 were predicted to be upregulated (exclusively identified on forward STR library), while 18 were downregulated (reverse CTR library; Table 2), indicating that, under these conditions, drought stress triggered an overall increase in gene expression. No common contigs were shared in forward and reverse libraries, confirming the accuracy of the suppression effect during library construction. This reinforces the relevance of SSHbased transcriptome analysis, even in the current NGS era, as a reliable and efficient approach to enrich and identify specific transcripts for given experimental contrasting conditions, in particular for orphan species such as A. magna, with no available transcriptome data. Contigs from both libraries were then ranked according to the number of contributing ESTs (reads per contig), and the 13 most expressed ones, including one unknown protein and 12 showing BLASTX identity with known abiotic-stress-responsive genes, were selected for validation by qRT-PCR (Table 3).

\section{Selection of Candidate Genes by qRT-PCR Analyses}

All 31 A. duranensis and 13 A. magna candidate genes analyzed by qRT-PCR showed specificity of transcript amplification, with high amplification efficiencies (Supplementary Table 1). Actin and ubiquintin previously identified by our group as the most stably expressed genes in $A$. duranensis and A. magna subjected to abiotic stress (Morgante et al. 2011) were used as reference genes. Their stable expression levels, regardless of tissue (root and leaf), treatment (control and stress), or collecting point, corroborate their herein usefulness, as well as the need to use at least two reference genes for qPCR data normalization.

Using pooled samples, the qRT-PCR expression pattern of the $31 \mathrm{~A}$. duranensis candidate genes was consistent with in silico analysis, although the latter showed less pronounced changes (Fig. 5; Table 1). These results confirmed the validity of estimating gene abundance using the gene count strategy (Beneventi et al. 2013) but reinforced the need for further validation through accurate in vitro analyses. Likewise, A. magna qRT-PCR expression profile data corroborated the subtracted cDNA libraries in silico analysis (Fig. 6; Table 3), as the 11 genes selected from STR library showed a predicted positive regulation, whereas the two genes derived from CTR library were downregulated.

The 13 selected TF genes are representatives of the most ubiquitous TF protein families in $A$. duranensis 454 transcripts
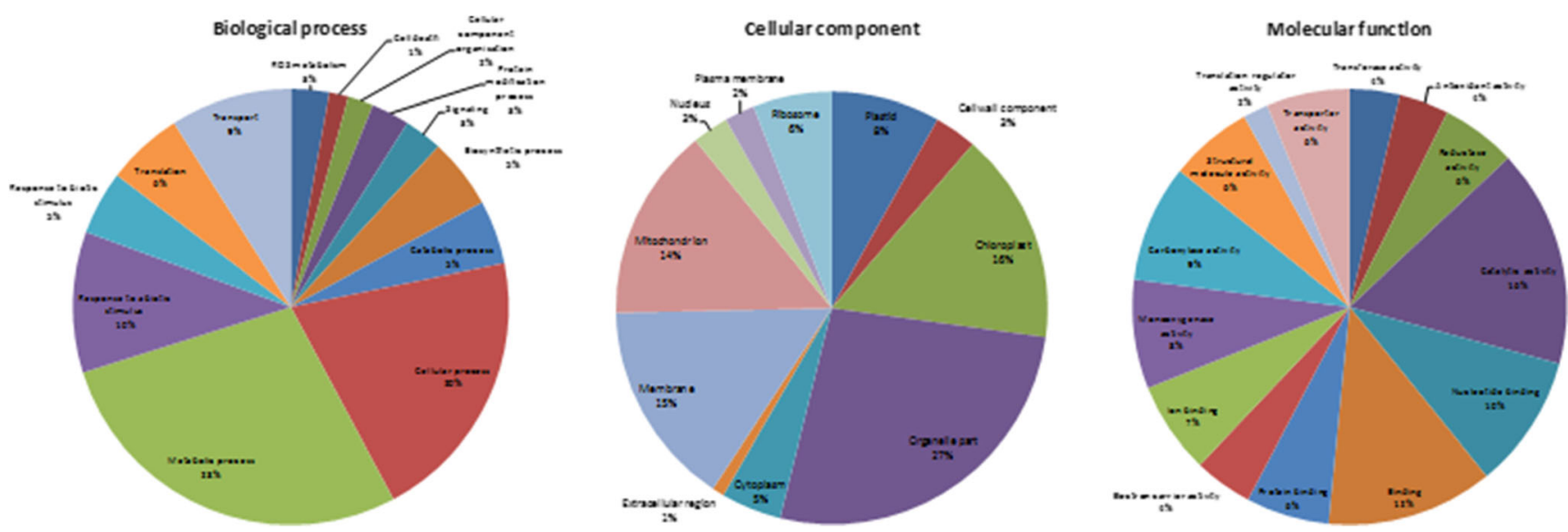

Fig. 4 Gene Ontology (GO) classification of the predicted A. magna unigenes according to biological process, cellular component, and molecular function using Blast2GO 


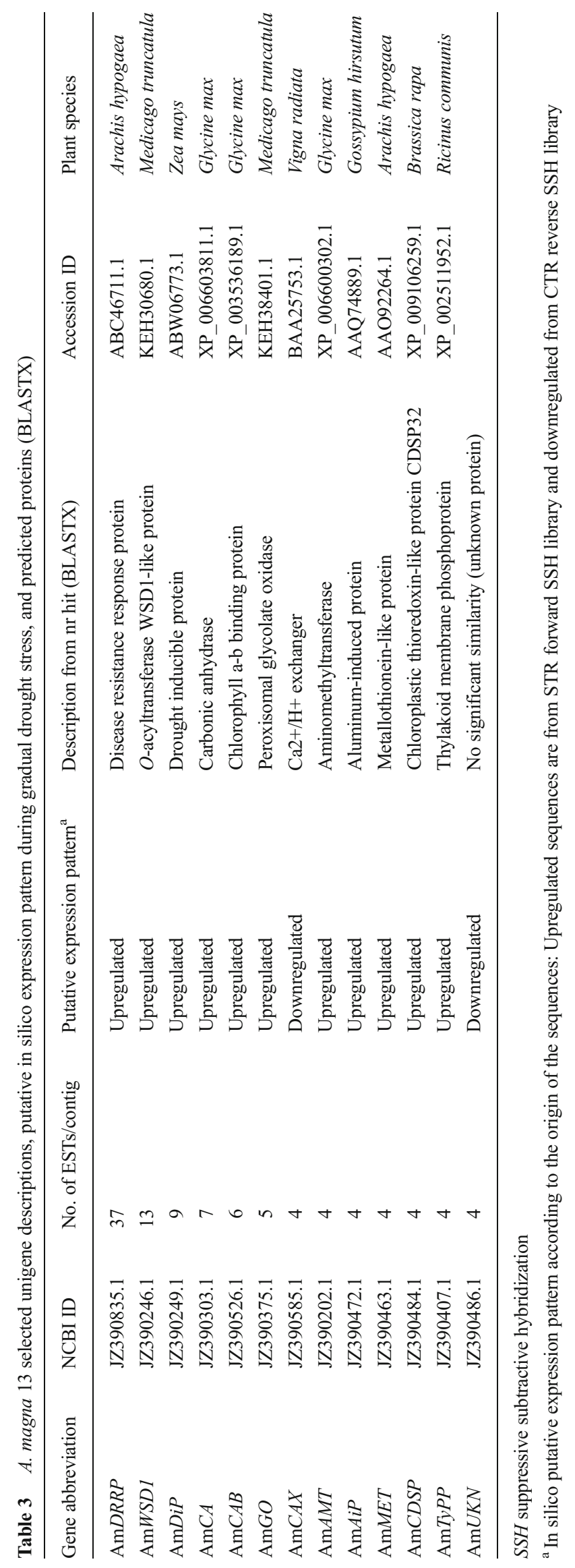




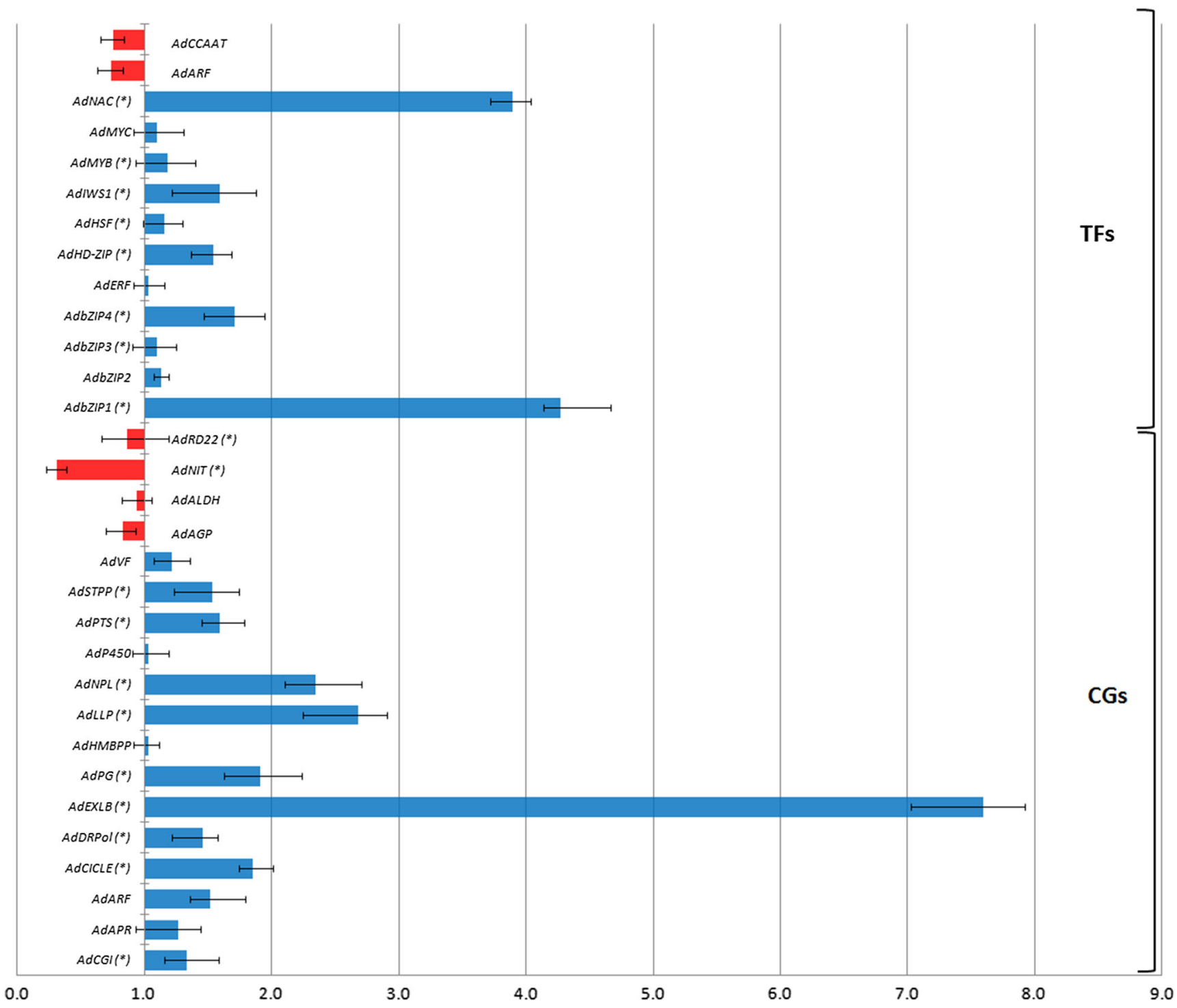

Fig. 5 Expression analysis of 13 transcription factors (TFs) and 18 other candidate genes (CGs) by qRT-PCR in $A$. duranensis plants submitted to a gradual water deficit. Axis $X$ represents the relative quantification (RQ)

between the DS and WW gathered samples, and the error bars indicate standard deviation of three replicates. Significantly $(p<0.05)$ upregulated or downregulated genes are indicated by asterisks

(Guimaraes et al. 2012) and are also referred to as potential candidates for drought adaptation improvement $(\mathrm{Hu}$ and Xiong 2014), such as bZIP, MYB, ERF, bHLH, $N A C$, and $H S F$. From those, eight showed significant upregulation under the dry-down according to qRT-PCR analysis (Fig. 5) with BZIP1 and NAC displaying the highest levels of expression (4.3- and 3.8-fold increase, respectively). $b Z I P$ and $N A C$ families are highly represented in our $454 \mathrm{~A}$. duranensis dataset as 13 and $7 \%$, respectively (Guimaraes et al. 2012), and also among the ten most represented TF families currently described in the peanut transcriptome (Jin et al. 2014). Under this perspective, A. duranensis bZIP1 and NAC candidates (Ad $b Z I P 1$ and $\operatorname{Ad} N A C$, respectively) were selected for a more comprehensive qRT-PCR analysis.
Besides TF genes, another $18 \mathrm{~A}$. duranensis candidate genes were also analyzed by qRT-PCR and 11 of them displayed a significant change in mRNA levels under drought imposition (Fig. 5). A gene coding for putative A. duranensis Expansin-like B ( $\operatorname{Ad} E X L B)$ displayed the highest (7.6-fold) levels of positive differential expression in DS plants compared to WW, whereas Nitrilase (AdNIT) which decreased nearly 2.3 -fold was the only candidate gene that showed a significant downregulation (Fig. 5). These genes are associated with water loss control and growth delay in response to drought perception (Ma et al. 2013; Piotrowski 2008) and were selected for further qRT-PCR evaluation.

Analysis by qRT-PCR of 13 candidate genes from A. magna showed that 11 of them displayed an upregulated profile in response to water deficit imposition while the 


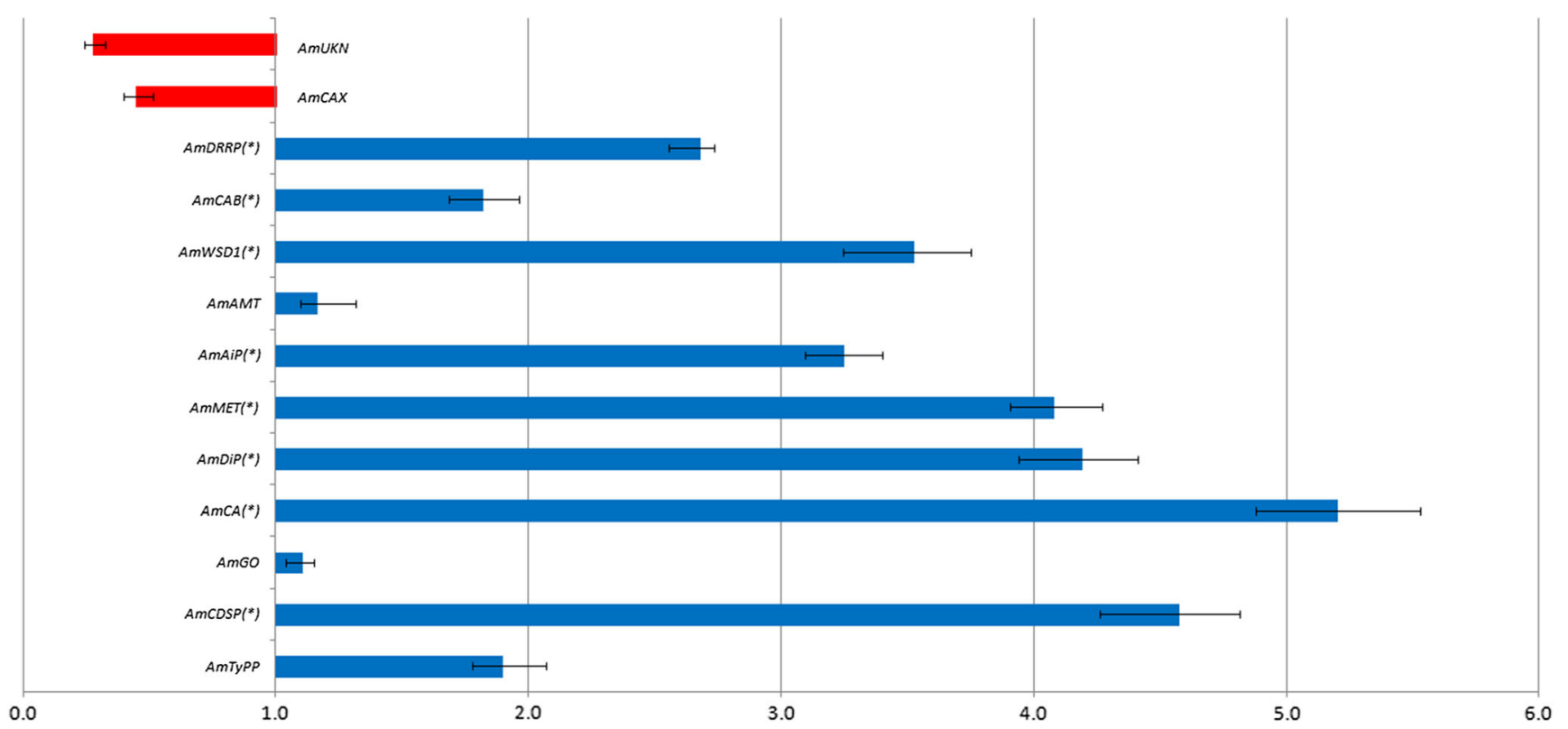

Fig. 6 Expression analysis of 13 candidate genes by qRT-PCR in A. magna plants submitted to a gradual water deficit. Axis $X$ represents the relative quantification (RQ) between the DS and WW gathered

remaining two were downregulated (Fig. 6). However, as also observed for $A$. duranensis, only a few candidate genes (DiP, $C D S P, M E T$, and $C A$ ) had a high (fold change $\geq 4$ ) and significant differential level of transcription. As their significant upregulation could be associated with drought tolerance mechanisms, these genes were also selected for qRT-PCR analysis.

\section{Detailed Expression Profile of Candidate Genes}

Additional qRT-PCR analysis was conducted to allow a detailed expression profiling for A. duranensis (AdbZIP1, $\operatorname{Ad} N A C, \operatorname{Ad} E X L B$, and $\operatorname{Ad} N I T)$ and A. magna (AmDiP, $\mathrm{AmCDSP}, \mathrm{Am} M E T$, and $\mathrm{AmCA}$ ) candidate genes. For this, root and leaf samples from $A$. duranensis and A. magna at each collecting point were analyzed and expression profiles evaluated along the gradual drought imposition.

The four primers designed for $A$. duranensis candidate genes showed high amplification efficiency (0.87 to 0.98 ) and a specific peak during melting curve analysis when using A. magna cDNA as template. Equally, A. magna primers efficiently amplified (0.84 to 0.95$) A$. duranensis samples and resulted in single-peaked melting curves. Moreover, all four A. duranensis genes presented at least one contig as orthologous in A. magna cDNA libraries and, as expected, the upregulated genes ( $\operatorname{Ad} b Z I P 1, \operatorname{Ad} N A C$, and $\operatorname{Ad} E X L B)$ in the STR library and the downregulated AdNIT in the CTR library. Similarly, all four A. magna genes (AmDiP, $\mathrm{AmCDSP}, \mathrm{Am} M E T$, and $\mathrm{AmCA}$ ) presented at least one orthologous contig, exclusively in the stressed $A$. duranensis 454 library. These high levels of similarity are expected as samples, and the error bars indicate standard deviation of three replicates. Significantly $(p<0.05)$ upregulated or downregulated genes are indicated by asterisks

previous findings showed an extensive synteny between AA (A. duranensis) and BB (A. magna) genomes of Arachis (Moretzsohn et al. 2009).

The eight candidate genes exhibited distinctive responses to drought challenge (Fig. 7), and in general, the results corroborated previous in silico and qRT-PCR analyses using pooled samples (collecting points gathered), demonstrating that pools can be a simpler and faster way to select highly expressed candidates among a large number of genes identified using in silico analysis.

Candidate gene expression behavior at different collecting points along the gradual water deficit was, on the whole, similar for both species, as well as during drought recovery steps (Fig. 7). In general, gene expression levels in roots were higher than in leaves, even for the genes selected from A. magna SSH leaf libraries. In fact, roots are one of the primary sites of drought perception, which triggers a cascade of gene expression responses, resulting in physiological changes and eventually determining the level of adaptation to the stress in the plant as a whole ( $\mathrm{Hu}$ and Xiong 2014). Therefore, roots were revealed to be a good target for the study of $A$. duranensis and A. magna genes involved in drought perception and key processes underlying plant adaptation and tolerance to water-limited conditions. Proteins coded by the eight candidates genes are involved in these processes by controlling functions related to gene expression regulation, primary metabolism and flux, hormone homeostasis, prevention of cell damage, and protection/adaptation of cell structures and might function to enhance drought adaptation and tolerance in $A$. duranensis and A. magna. 

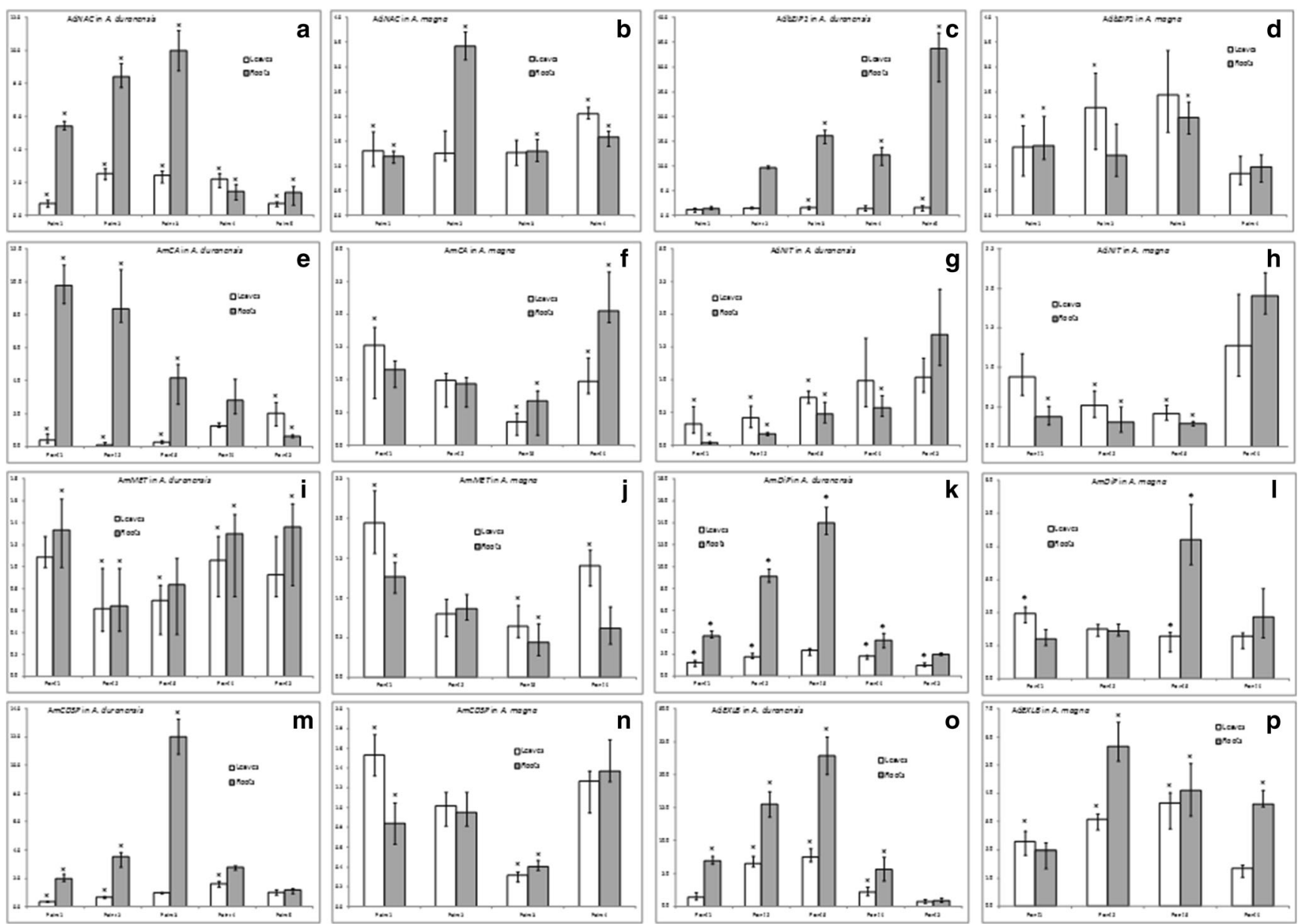

Fig. 7 a-p Expression analysis of eight candidate genes by qRT-PCR in leaves and roots of $A$. duranensis and A. magna submitted to a gradual water deficit and rehydration, at different collecting points. Axis $X$
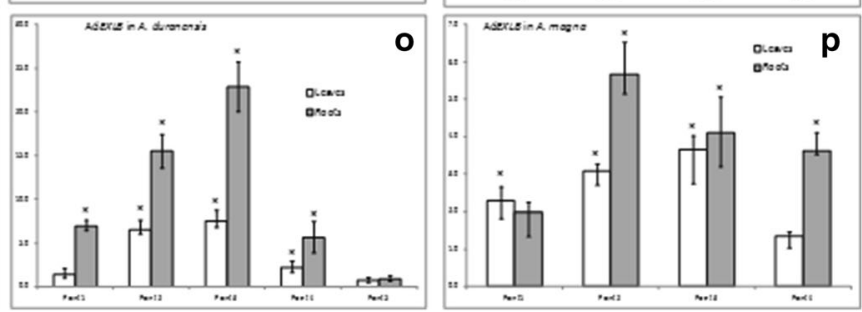

represents the relative quantification (RQ) between the DS and WW pools and the mean value of three replicates. Significantly $(p<0.05)$ upregulated or downregulated genes are indicated by asterisks

\section{Genes Involved in Signal Transduction}

Ad $N A C$ exhibited a significant and gradual enhancement in transcript levels in A. duranensis leaves and roots, up to rehydration point 4 , when its expression dropped to near the basal level (Fig. 7a). Particularly in roots, this gene reached higher levels of upregulation (10-fold) at the threshold collecting point of the water deficit treatment (point $3=$ NTR 0.28 ; Fig. 7a), when evident root desiccation was observed (Fig. 2b). Conversely, a discreet increase in $\operatorname{Ad} N A C$ transcripts was observed in A. magna, with levels close to 1.2-fold during stress imposition, excepted at point 2 , when expression in roots showed a significant upregulation of 3.41-fold (Fig. 7b). After rehydration, AdNAC expression slightly increased in A. magna roots and leaves (1.59- and 2.05-fold, respectively). $N A C$ is a multifunctional plant-specific TF family, with more than 8000 putative members (Jin et al. 2014), and is involved in regulation of various stress signaling responses, conferring tolerance to abiotic stress in transgenic plants (Puranik et al. 2012). Many studies showed that the overexpression of $N A C$-coding genes could improve drought

tolerance and that they are more effective when under the control of stress-responsive or tissue-specific promoters $(\mathrm{Hu}$ and Xiong 2014; Nakashima et al. 2014). Moreover, the overexpression of a peanut $N A C$ gene in Arabidopsis conferred enhanced tolerance to drought and salinity under greenhouse conditions (Liu et al. 2011), corroborating its potential role in signaling stress responses by improving drought tolerance.

Another TF gene analyzed here, AdbZIP1, was also remarkably responsive to the water deficit treatment in A. duranensis roots, with its expression rising 16-fold from point 1 to 3 (Fig. 7c) and peaking at $72 \mathrm{~h}$ after rehydration, with an upregulation of 33.69-fold. On the other hand, its expression in A. duranensis leaves and in A. magna roots and leaves seemed to be not, or very weakly, regulated in response to drought or to rehydration, with relative expression ranging from 0.85 - to 2.44 -fold (Fig. 7c, d). These expression profiles indicated that AdbZIP1 is highly involved, at least in A. duranensis roots, in the positive modulation of downstream signaling pathways of the drought recovery process and, to a lesser extent, in the response to the water deficit. Members of bZIP1 TFs, such as AREBs/ABFs, regulate the expression of 
many genes in the abscisic acid (ABA)-dependent signaling pathway in response to drought and dehydration conditions (Hu and Xiong 2014; Nakashima et al. 2014). Recent studies demonstrated that the expression of an AREB gene isolated from peanut modulated reactive oxygen species (ROS) accumulation and endogenous ABA levels in transgenic Arabidopsis thaliana, with improved drought tolerance ( $\mathrm{Li}$ et al. 2013).

Interestingly, it was demonstrated that members of both $\mathrm{TF}$ families here analyzed, $b Z I P 1$ and $N A C$, interact synergistically to control water loss, helping plants to survive under dehydration or osmotic stress conditions (Xu et al. 2013). This observation suggests the cooperative and complex interactions between $b Z I P 1$ and $N A C$ regulons. Therefore, the increased expression of $\operatorname{AdNAC}$ and $\operatorname{Ad} b Z I P 1$ in $A$. duranensis roots in a putative cooperative interaction in response to the imposed gradual water deficit may indicate that these genes are promising candidates for engineering drought tolerance in peanut.

\section{Genes Involved in Primary Metabolism}

In $A$. duranensis, $\mathrm{AmCA}$ gene showed an opposite profile in roots compared to leaves (Fig. 7e): when an evident elevation (9.79-fold at collecting point 1) of AmCA transcript levels was observed in roots, a remarkable suppression of its levels occurred in leaf tissues (10-fold downregulation at collecting point 2). After re-watering, AmCA expression in both tissues returned to the basal levels. However, in A. magna, AmCA showed a slight upregulation, followed by downregulation in response to drought stress which was recovered after rehydration (Fig. 7f). Carbonic anhydrase $(C A)$ is an ubiquitous zinccontaining enzyme which catalyzes the reversible hydration of $\mathrm{CO}_{2}$ and has a putative role in the decreasing of photosynthesis under drought conditions (Perez-Martin et al. 2014). Its expression under dehydration and osmotic stress could be species-dependent or even associated with its subcellular localization (Wu et al. 2012). A decrease in $C A$ expression during drought stress, followed by an upregulation after rehydration, suggested the involvement of this gene in the regulation of mesophyll conductance to $\mathrm{CO}_{2}$ (Perez-Martin et al. 2014), by altering carbon primary metabolism. A negative modulation of AmCA expression in both wild Arachis along the low water availability is, hence, in accordance with the literature. Nevertheless, the opposite behavior of $\mathrm{AmCA}$ in $A$. duranensis leaves and roots is a very interesting issue concerning its tissue-specific expression that will be addressed in forthcoming studies.

AdNIT showed the strongest repression levels in response to dry-down imposition in both $A$. duranensis and A. magna (Fig. $7 \mathrm{~g}, \mathrm{~h}$ ). Remarkably in A. duranensis roots, transcripts displayed a drastic and significant decrease (25-fold) during initial dry-down stages, as an immediate reaction to drought stress perception (Fig. 7g). As stress progressed, a gradual increase in the expression was observed, achieving a positive regulation (1.69-fold) $72 \mathrm{~h}$ after rehydration (point 5; Fig. $7 \mathrm{~g}$ ). In a similar way, the levels of $\operatorname{AdNIT}$ transcripts in leaves of A. duranensis significantly decreased (3-fold) at the beginning of stress and gradually recovered its basal level $30 \mathrm{~min}$ after re-watering (point 4; Fig. 7g). In A. magna samples, the repression of AdNIT expression as a possible stress perception seemed to be delayed comparing to A. duranensis (Fig. 7g, h), showing a gradual and significant expression decline as the water availability in the soil decreased and reaching the lowest level of expression (2.38- and 3.45-fold for leaves and roots, respectively) at the end of stress (point 3; Fig. 7h). Nitrilaselike proteins (NIT) are plant enzymes that hydrolyze organic nitriles to ammonia and corresponding carboxylic acids. NIT is involved in cyanide detoxification and nitrogen recycling and also in biosynthesis of the plant hormone indole-3-acetic acid (IAA), the levels of which could decline in response to drought perception causing growth delay (Piotrowski 2008). The downregulation of AdNIT in response to water deficit stimulus could be associated with the putative role of this enzyme in processes regulating cell proliferation and differentiation in order to maintain a balance with plant cell death (PCD) and also in cyanide detoxification and nitrogen primary metabolism (Doskocilova et al. 2013; Piotrowski 2008). Curiously, NIT interacts with ERF and NAC proteins, which are TF family members involved in the regulation of ethylene and IAA responses as well as in drought stress tolerance (Huh et al. 2012; Xu et al. 1998), as discussed above. The overexpression of Arabidopsis NIT1 resulted in the suppression of cell growth and increased levels of PCD and, conversely, plants with reduced NIT1-3 expression of showed diminution of leaf and root growth (Doskocilova et al. 2013). Those results indicate that the inhibition of NIT expression trigged by water-limited conditions could help plants in controlling cell growth and root elongation to circumvent drought adverse effects. However, the specific role of NIT in drought responses, involving plant developmental processes, such as cytokinesis and PCD and IAA and ethylene homeostasis, remains to be demonstrated.

\section{Genes Involved in Cell Protection and Adaptive Mechanisms}

AmMET exhibited overall similar responses to drought in leaves and roots for both A. duranensis and A. magna (Fig. 7i, j). In these species, AmMET apparently responds at the beginning of the stress with a modest increase in its expression that changed to a slight downregulation along the treatment followed by the increasing expression to basal levels after rehydration (Fig. 7i, j). Metallothioneins (MET) are metal-binding cytosolic proteins widely distributed in living organisms and implicated in ROS scavenging and metal detoxification homeostasis to protect cells against a variety of 
stresses (Leszczyszyn et al. 2013). AdMET codes for a putative MET type 3 that is thought to be positively regulated in response to water-limited conditions and thereby plays a role in drought tolerance (Xue et al. 2009), probably by protecting cells and reducing ROS damage during defense signaling. Therefore, the herein AdMET positive regulation, previously indicated by in silico and qRT-PCR analyses conducted with A. magna gathered samples (Table 3 and Fig. 6, respectively), occurred indeed only at the beginning of the stress (point 1) in both $A$. duranensis and A. magna samples, as demonstrated in the point-by-point qRT-PCR analysis (Fig. 7i, j).

The other $A$. magna candidate gene, AmDiP, displayed a marked increase in transcript level in $A$. duranensis roots, with the peak (14.01-fold) at the threshold collecting point of the treatment, followed by a decrease to basal levels $72 \mathrm{~h}$ after rehydration (Fig. 7k). AmDiP presented a proportionate expression pattern in $A$. duranensis leaves, but with lower levels than in roots (Fig. 7k). For A. magna, the upregulation peak of AmDiP (4.20-fold) in roots was at the threshold point of the treatment, while in the other root collecting points and in leaves, this gene expression remained close to the basal level (1.21 - to 1.98-fold) (Fig. 71). Drought-induced proteins (DiP) are small proteins present in several plant species, whose expression is highly modulated by abiotic stress (Kalifa et al. 2004). AmDiP has high sequence similarity with a group of DiP plant proteins that have the same expression profile as the one herein determined for Arachis spp., i.e., highly activated under water-limited conditions. DiPs are regulated by an ABA-dependent pathway whenever the plant is in a stressful environment, and it is associated with chromatin protection or signal transduction (Kalifa et al. 2004), but its biological function remains unclear.

AmCDSP exhibited a very similar expression profile to $\mathrm{AmDiP}$ in $A$. duranensis roots, with an intense increase in transcript levels in response to drought imposition (peak of 12.02-fold at threshold point) and basal expression return during rehydration (Fig. $7 \mathrm{~m}$ ). Conversely, expression of AmCDSP in $A$. duranensis leaves displayed a significant decline (2.94-fold) at the drought beginning, followed by a gradual recovery to basal levels just after re-watering which was maintained (Fig. 7m). In A. magna, AmCDSP showed a different expression behavior to $A$. duranensis, with a gradual expression decline during drought imposition (3.14- and 2.47fold, for leaves and roots, respectively) at the threshold stress collecting point (Fig. 7n). After 5 h of rehydration, AmCDSP expression recovered to the basal levels in both tissues. CDSP gene codes for Chloroplast Drought-induced Stress Protein described as highly responsive to drought, but with no attributed function (Broin et al. 2000). AmCDSP showed a high degree of similarity to the $A$. thaliana thioredoxin-like protein CDSP32 which is supposed to participate in the prevention of cell damage and protection of chloroplast structures upon oxidative stress within the chloroplast during water deficit (Broin et al. 2000). This protective function could be extended to AmCDSP since its expression pattern observed in A. duranensis roots is in accordance with CDSP32 previously described behavior under drought stress.

Notably, among these eight selected candidates, $\operatorname{Ad} E X L B$ showed the highest transcriptional induction in response to the progressive water deficit in soil (Fig. 7). Although at different magnitude, its expression profile in A. duranensis roots and leaves was very similar to $A$. magna, with a strong induction in response to drought stress followed by significant decrease after rehydration (Fig. 7o, p). In A. duranensis, $\operatorname{Ad} E X L B$ expression reached the maximum level of induction (22.87- and 7.54-fold in roots and leaves, respectively) at the threshold collecting point of the stress treatment (point 3; Fig. 7o). Upon $72 \mathrm{~h}$ of rehydration, its transcriptional levels in DS plants decreased drastically, recovering to its basal level, i.e., similar to WW plants (Fig. 7o). In A. magna, AdEXLB expression showed the same behavior, but its expression peak occurred at point 2 in roots and at point 3 in leaves (5.68-fold and 3.65-fold, respectively; Fig. 7p). After re-watering, expression in leaves decreased to basal levels, whereas in roots, it maintained a similar expression (3.63-fold) to the threshold collecting point of the stress treatment. This suggests that roots need more than $5 \mathrm{~h}$ of rehydration to recover $\operatorname{Ad} E X L B$ basal expression. $E X L B$ gene is a member of the Expansin superfamily of plant cell wall-loosening proteins involved in many developmental processes as well as in adaptive responses to mechanical or environmental stimuli. The involvement of genes from the Expansin superfamily in cell wall plasticity is associated with the control of water loss, probably promoting cell wall relaxation aiming cell turgor adjustment, as shown in transgenic drought-tolerant plants obtained by overexpressing Expansin-coding genes (Li et al. 2011; Lü et al. 2013; Ma et al. 2013). Studies conducted in many plants, including the resurrection plant Craterostigma plantagineum, also showed increasing expression of Expansins at early stages of dehydration (Choi et al. 2006). Recent genomewide characterization of Expansin genes in plants revealed that the Expansin like-B (EXLB) gene here identified is not well characterized and, together with Expansin likeA, one of the less represented member of the Expansin superfamily (Zhu et al. 2014). Since EXLB amino acid sequences are very divergent in plants, it was suggested that its action mode could differ from that of other superfamily members. Moreover, our preliminary studies indicated that the increase in $\operatorname{Ad} E X L B$ expression could be associated with more conservative transpiration behavior in nine wild Arachis species submitted to water-limited conditions, when compared with cultivated genotypes (not published). Therefore, EXLB became a potential target for the enhancement of drought tolerance in transgenic plants through cell dehydration control via turgor upholding and further analysis is underway. 


\section{Conclusions}

Drought stress in plants is a complex issue and often involves an intricate chain orchestrated by physiological events that affect transcriptional and translational regulation in plants. Different plant genotypes often harbor diverse mechanisms of response and adaptation to drought, which might include the expression of a distinct set of genes that trigger changes in specific biochemical pathways and structural arrangements. Wild Arachis species (A. duranensis and A. magna) potentially harbor improved performance under abiotic stress since their native areas are water-limited environments, making them interesting genotypes for drought-related gene discovery. In order to better address the multiplicity of biological processes involved in drought perception and responses, the transcriptome profile of these two wild species was analyzed at different stages of a gradual water deficit, followed by rehydration. Regardless of the differences in the methodological conception in the construction and sequencing of the libraries and differences between $\mathrm{AA}$ (A. duranensis) and $\mathrm{BB}$ (A. magna) genomes, these two species have a high number of common genes identified as differentially expressed, suggesting a similar transcriptional modulation in response to water deficit. Moreover, from those, eight candidate genes shared similar expression profiles in response to drought challenge and recovery. This included genes involved in signal transduction, which were highly induced by drought perception or after rehydration in $A$. duranensis roots, such as NAC and $b Z I P 1$. Other genes that better enable plants to survive under stress conditions, including those involved in the adaptation of primary metabolism, such as CA or NIT, or coding for proteins implicated in cell protection or adaptation mechanisms, like $C D S P, D i P$, or $E X L B$, were also identified in both A. duranensis and A. magna. This enabled the unraveling of novel promising candidates that might enhance drought tolerance by gene manipulation in bio-engineered crops, particularly peanut and other legumes.

Despite recent efforts of the peanut scientific community, the extension of Arachis genomic resources is still limited, hindering Arachis gene expression dynamics characterization. Thus, besides a valuable resource for gene discovery, this transcriptome survey of wild Arachis species also contributes to a better understanding of gene modulation in response to water deficit and rehydration, providing new tools for peanut breeding programs, such as the characterization of new alleles and development of molecular markers associated with drought stress.

Acknowledgments The authors gratefully acknowledge host institutions, CNPq, CAPES, FAP-DF, The Challenge Program Generation, and Tropical Legume Improvement (TL1) for funding this research.

Authors' Contributions Conceived and designed the experiments: ACMB, ACGA, SCMLB, and PMG; performed the experiments:
CVM, CMRS, MAPS, AKS, and ACQM; analyzed the data: ACMB, CVM, ACGA, CCV, OB, and RCT; wrote the paper: ACMB, ACGA, SCMLB, and PMG; contributed reagents/materials/analysis tools: ACMB, ACGA, SCMLB, DJB, and PMG; and critically revised the manuscript: all authors.

Open Access This article is distributed under the terms of the Creative Commons Attribution 4.0 International License (http:// creativecommons.org/licenses/by/4.0/), which permits unrestricted use, distribution, and reproduction in any medium, provided you give appropriate credit to the original author(s) and the source, provide a link to the Creative Commons license, and indicate if changes were made.

\section{References}

Altschul SF, Gish W, Miller W, Myers EW, Lipman DJ (1990) Basic local alignment search tool. J Mol Biol 215:403-410

Beneventi M et al (2013) Transcription profile of soybean-root-knot nematode interaction reveals a key role of phythormones in the resistance reaction. BMC Genomics 14:322

Bertioli DJ, Seijo G, Freitas FO, Valls JFM, Leal-Bertioli SCM, Moretzsohn MC (2011) An overview of peanut and its wild relatives. Plant Genet Resour 9:134-149

Brasileiro ACM, Araujo ACG, Leal-Bertioli SC, Guimarães PM (2014) Genomics and genetic transformation in Arachis. Int J Plant Biol Res 2:1017

Broin M, Cuine S, Peltier G, Rey P (2000) Involvement of CDSP 32, a drought-induced thioredoxin, in the response to oxidative stress in potato plants. FEBS Lett 467:245-248

Chen X et al (2013) Deep sequencing analysis of the transcriptomes of peanut aerial and subterranean young pods identifies candidate genes related to early embryo abortion. Plant Biotechnol J 11:115127

Choi D, Cho H-T, Lee Y (2006) Expansins: expanding importance in plant growth and development. Physiol Plant 126:511-518

Ding H, Zhang ZM, Qin FF, Dai LX, Li CJ, Ci DW, Song WW (2014) Isolation and characterization of drought-responsive genes from peanut roots by suppression subtractive hybridization. Electron $\mathrm{J}$ Biotechnol 17:304-3010

Doskocilova A et al (2013) Nitrilase1 regulates the exit from proliferation, genome stability and plant development. New Phytol 198:685698

Gotz S et al (2008) High-throughput functional annotation and data mining with the Blast2GO suite. Nucleic Acids Res 36:3420-3435

Govind G, Thamme Gowda HV, Kalaiarasi PJ, Iyer DR, Muthappa SK, Nese S, Makarla UK (2009) Identification and functional validation of a unique set of drought induced genes preferentially expressed in response to gradual water stress in peanut. Mol Genet Genomics 281:591-605

Guimaraes P et al (2012) Global transcriptome analysis of two wild relatives of peanut under drought and fungi infection. BMC Genomics $13: 387$

Guo BZ et al (2008) Peanut gene expression profiling in developing seeds at different reproduction stages during Aspergillus parasiticus infection. BMC Dev Biol 8:12

Holbrook CC, Ozias-Akins P, Chu Y, Guo B (2011) Impact of molecular genetic research on peanut cultivar development. Agronomy 1:3-17

$\mathrm{Hu} \mathrm{H}$, Xiong L (2014) Genetic engineering and breeding of droughtresistant crops. Ann Rev Plant Biol 65:715-741 
Huh SU, Lee S-B, Kim HH, Paek K-H (2012) ATAF2, a NAC transcription factor, binds to the promoter and regulates NIT2 gene expression involved in auxin biosynthesis. Mol Cells 34:305-313

Jain M (2012) Next-generation sequencing technologies for gene expression profiling in plants. Brief Funct Genomics 11:63-70

Jain AK, Basha SM, Holbrook CC (2001) Identification of droughtresponsive transcripts in peanut (Arachis hypogaea L.). Electron J Biotechnol 4:59-67

Jin J, Zhang H, Kong L, Gao G, Luo J (2014) PlantTFDB 3.0: a portal for the functional and evolutionary study of plant transcription factors. Nucleic Acids Res 42:D1182-D1187

Juenger TE (2013) Natural variation and genetic constraints on drought tolerance. Curr Opin Plant Biol 16:1-8

Kalifa Y, Gilad A, Konrad Z, Zaccai M, Scolnik P, Bar-Zvi D (2004) The water-and salt-stress-regulated Asrl (abscisic acid stress ripening) gene encodes a zinc-dependent DNA-binding protein. Biochem J 381:373-378

Krapovickas A, Gregory WC (2007) Taxonomy of the genus Arachis (Leguminosae). Bonplandia 16(Suppl):1-205

Leal-Bertioli SCM et al (2009) Identification of candidate genome regions controlling disease resistance in Arachis. BMC Plant Biol 9: 112

Leal-Bertioli SCM et al (2012) The effect of tetraploidization of wild Arachis on leaf morphology and other drought-related traits. Environ Exp Bot 84:17-24

Leszczyszyn OI, Imam HT, Blindauer CA (2013) Diversity and distribution of plant metallothioneins: a review of structure, properties and functions. Metallomics 5:1146-1169

Li F et al (2011) Drought tolerance through over-expression of the expansin gene TaEXPB23 in transgenic tobacco. J Plant Physiol 168:960-966

Li J, Dai X, Liu T, Zhao PX (2012) LegumeIP: an integrative database for comparative genomics and transcriptomics of model legumes. Nucleic Acids Res 40:D1221-D1229

Li X-Y et al (2013) Overexpression of Arachis hypogaea AREB1 gene enhances drought tolerance by modulating ROS scavenging and maintaining endogenous ABA content. Int J Mol Sci 14:1282712842

Li X, Lu J, Liu S, Liu X, Lin Y, Li L (2014) Identification of rapidly induced genes in the response of peanut (Arachis hypogaea) to water deficit and abscisic acid. BMC Biotechnol 14:58

Liu X, Hong L, Li X-Y, Yao Y, Hu B, Li L (2011) Improved drought and salt tolerance in transgenic Arabidopsis overexpressing a NAC Transcriptional Factor from Arachis hypogaea. Biosci Biotech Bioch 75:443-450

Lü P, Kang M, Jiang X, Dai F, Gao J, Zhang C (2013) RhEXPA4, a rose expansin gene, modulates leaf growth and confers drought and salt tolerance to Arabidopsis. Planta 237:1547-1559

Luo M, Liang XQ, Dang P, Holbrook CC, Bausher MG, Lee RD, Guo BZ (2005) Microarray-based screening of differentially expressed genes in peanut in response to Aspergillus parasiticus infection and drought stress. Plant Sci 169:695-703

Ma N, Wang Y, Qiu S, Kang Z, Che S, Wang G, Huang J (2013) Overexpression of OsEXPA8, a root-specific gene, improves rice growth and root system architecture by facilitating cell extension. PLoS ONE 8:e75997

Madlung A, Wendel JF (2013) Genetic and epigenetic aspects of polyploid evolution in plants. Cytogenet Genome Res 140:270-285

Moretzsohn MC et al (2005) A microsatellite-based, gene-rich linkage map for the AA genome of Arachis (Fabaceae). Theor Appl Genet 111:1060-1071

Moretzsohn MC et al (2009) A linkage map for the B-genome of Arachis (Fabaceae) and its synteny to the A-genome. BMC Plant Biol 9:40

Morgante CV, Guimaraes PM, Martins AQ, Araujo ACG, Leal-Bertioli SCM, Bertioli DJ, Brasileiro ACM (2011) Reference genes for quantitative reverse transcription-polymerase chain reaction expression studies in wild and cultivated peanut. BMC Res Notes 4:339

Morgante CV et al (2013) A survey of genes involved in Arachis stenosperma resistance to Meloidogyne arenaria race 1. Funct Plant Biol 40:1298-1309

Muchow RC, Sinclair TR (1991) Water deficit effects on maize yields modeled under current and "greenhouse" climates. Agron J 83: 1052-1059

Nakashima K, Yamaguchi-Shinozaki K, Shinozaki K (2014) The transcriptional regulatory network in the drought response and its crosstalk in abiotic stress responses including drought, cold, and heat. Front Plant Sci 5:170

Pandey MK et al (2012) Advances in Arachis genomics for peanut improvement. Biotech Adv 30:639-651

Perez-Martin A, Michelazzo C, Torres-Ruiz JM, Flexas J, Fernández JE, Sebastiani L, Diaz-Espejo A (2014) Regulation of photosynthesis and stomatal and mesophyll conductance under water stress and recovery in olive trees: correlation with gene expression of carbonic anhydrase and aquaporins. J Exp Bot 65:3143-3156

Pfaffl MW, Horgan GW, Dempfle L (2002) Relative expression software tool (REST (c)) for group-wise comparison and statistical analysis of relative expression results in real-time PCR. Nucleic Acids Res 30: 10

Piotrowski M (2008) Primary or secondary? Versatile nitrilases in plant metabolism. Phytochemistry 69:2655-2667

Proite K, Leal-Bertioli SCM, Bertioli DJ, Moretzsohn MC, da Silva FR, Martins NF, Guimaraes PM (2007) ESTs from a wild Arachis species for gene discovery and marker development. BMC Plant Biol 7:

Puranik S, Sahu PP, Srivastava PS, Prasad M (2012) NAC proteins: regulation and role in stress tolerance. Trends Plant Sci 17:369-381

Ranganayakulu G, Chandraobulreddy P, Thippeswamy M, Veeranagamallaiah G, Sudhakar C (2011) Identification of drought stress-responsive genes from drought-tolerant groundnut cultivar (Arachis hypogaea L. cv K-134) through analysis of subtracted expressed sequence tags. Acta Physiol Plant 34:361-377

Robledo G, Lavia GI, Seijo G (2009) Species relations among wild Arachis species with the A genome as revealed by FISH mapping of rDNA loci and heterochromatin detection. Theor Appl Genet 118: 1295-1307

Sawal RK, Ratan R, Yadav SBS (2004) Mesquite (Prosopis juliflora) pods as a feed resource for livestock - a review. Asian Australas J Anim Sci 17:719-725

Sinclair TR, Ludlow MM (1986) Influence of soil water supply on the plant water balance of four tropical grain Legumes. Aust J Plant Physiol 13:329-341

Telles GP, Silva FR (2001) Trimming and clustering sugarcane ESTs. Genet Mol Biol 24:17-23

Tirumalaraju SV, Jain M, Gallo M (2011) Differential gene expression in roots of nematode-resistant and -susceptible peanut (Arachis hypogaea) cultivars in response to early stages of peanut root-knot nematode (Meloidogyne arenaria) parasitization. J Plant Physiol $168: 481-492$

Volkov V, Amtmann A (2006) Thellungiella halophila, a salt-tolerant relative of Arabidopsis thaliana, has specific root ion-channel features supporting $\mathrm{K}+/ \mathrm{Na}+$ homeostasis under salinity stress. Plant $\mathrm{J}$ $48: 342-353$

Wu Y, Vreugdenhil D, Liu C, Fu W (2012) Expression of carbonic anhydrase genes under dehydration and osmotic stress in Arabidopsis thaliana leaves. Adv Sci Lett 17:261-265

$\mathrm{Wu} \mathrm{N}$ et al (2013) De novo next-generation sequencing, assembling and annotation of Arachis hypogaea L. Spanish botanical type whole plant transcriptome. Theor Appl Genet 126:1145-1149

$\mathrm{Xu} \mathrm{P}$ et al (1998) A nitrilase-like protein interacts with GCC box DNAbinding proteins involved in ethylene and defense responses. Plant Physiol 118:867-874 
Xu Z-Y et al (2013) The Arabidopsis NAC transcription factor ANAC096 cooperates with bZIP-type transcription factors in dehydration and osmotic stress responses. Plant Cell Online 25:4708-4724

Xue T, Li X, Zhu W, Wu C, Yang G, Zheng C (2009) Cotton metallothionein GhMT3a, a reactive oxygen species scavenger, increased tolerance against abiotic stress in transgenic tobacco and yeast. J Exp Bot 60:339-349

Yin D et al (2013) De novo assembly of the peanut (Arachis hypogaea L.) seed transcriptome revealed candidate unigenes for oil accumulation pathways. PLoS ONE 8:e73767

Zaman-Allah M, Jenkinson DM, Vadez V (2011) Chickpea genotypes contrasting for seed yield under terminal drought stress in the field differ for traits related to the control of water use. Funct Plant Biol $38: 270-281$
Zhang J et al (2012) De novo assembly and characterisation of the transcriptome during seed development, and generation of genic-SSR markers in peanut (Arachis hypogaea L.). BMC Genomics 13:90

Zhao S, Fernald RD (2005) Comprehensive algorithm for quantitative real-time polymerase chain reaction. J Comput Biol 12:1047-1064

Zhou Y, Gao F, Liu R, Feng J, Li H (2012) De novo sequencing and analysis of root transcriptome using 454 pyrosequencing to discover putative genes associated with drought tolerance in Ammopiptanthus mongolicus. BMC Genomics 13:266

Zhu Y, Wu N, Song W, Yin G, Qin Y, Yan Y, Hu Y (2014) Soybean (Glycine max) expansin gene superfamily origins: segmental and tandem duplication events followed by divergent selection among subfamilies. BMC Plant Biol 14:93 